\title{
Reversing Conditional Orderings
}

\author{
Rachele Foschi, ${ }^{*}$ Fabio Spizzichino ${ }^{\dagger}$
}

\begin{abstract}
We analyze some specific aspects concerning conditional orderings and relations among them. To this purpose we define a suitable concept of reversed conditional ordering and prove some related results. In particular we aim to compare the univariate stochastic orderings $\leq_{\text {st }}$, $\leq_{\mathrm{hr}}$, and $\leq_{\mathrm{lr}}$ in terms of differences among different notions of conditional orderings. Some applications of our result to the analysis of positive dependence will be detailed. We concentrate attention to the case of a pair of scalar random variables $X, Y$. Suitable extensions to multivariate cases are possible.
\end{abstract}

Keywords: Positive dependence, reversed conditional orderings, default contagion, conditional independence.

\section{Introduction}

Stochastic orderings between random variables (or random vectors) constitute primary tools for the description and the characterization of concepts of stochastic dependence. On the one hand the relevant literature in this direction is very well established (see in particular [17] and the references contained therein). On the other hand it still continues to offer various suggestions for interesting work.

Here we consider stochastic orderings between (one-dimensional) conditional distributions, also called conditional orderings. We will analyze some specific aspects concerning such orderings and relations among them.

Specially in a statistical setting, the following problem is of interest: what can be said about dependence of $X$ w.r.t. $Y$ (where $X$ and $Y$ are random

*rachele.foschiimtlucca.it, Economics and Institutional Change Research Area, IMT Advanced Studies Lucca, Via Elisa, 63, 55100 Lucca, Italy

†fabio.spizzichino@uniroma1.it, Dipartimento di Matematica G. Castelnuovo, Università degli Studi di Roma La Sapienza, Piazzale Aldo Moro 5, 00185 Rome, Italy 
variables or random vectors) when we assume that $Y$ is stochastically increasing w.r.t. $X$ in some specified sense? Attention to this topic has been given several times in the literature under different stand-points or different languages. One can see in particular the basic paper [7], [19, Chapter 3] and the recent papers $[5,6]$. Related to this theme one can also see [15].

Some detailed aspects of this theme will be considered here for the special case of two scalar random variables. As a motivating purpose, we aim to compare the univariate stochastic orderings $\leq_{\mathrm{st}}, \leq_{\mathrm{hr}}$, and $\leq_{\mathrm{lr}}$ in terms of differences among notions of conditional orderings.

For scalar random variables $X$ and $Y$, we consider different conditional orderings of the form

$$
\mathcal{L}(Y \mid X \in I) \leq_{*} \mathcal{L}\left(Y \mid X \in I^{\prime}\right)
$$

where $I, I^{\prime}$ are intervals of different types and $\leq_{*}$ stands for $\leq_{\mathrm{st}}, \leq_{\mathrm{hr}}$, or $\leq_{\mathrm{lr}}$. In a few words, we can summarize our work by saying that we analyze implications or equivalences concerning such relations. Along this direction we will show some simple results that, at the best of our knowledge, have not been pointed out so far.

A concept of reversed conditional ordering will in particular emerge as natural from our discussion and our results will point out some symmetries existing between the mentioned univariate stochastic orderings $\leq_{\mathrm{st}}, \leq_{\mathrm{hr}}, \leq_{\mathrm{lr}}$ and different types of conditional orderings (where a "type" of ordering can be defined in terms of the possible choices for the intervals $I, I^{\prime}$ appearing in (1)). A main result in this direction is Theorem 1 .

It is clear that conditional orderings define special notions of positive dependence (see in particular [4] and references cited therein). In the article, we will analyze positive dependence properties corresponding to the considered conditional orderings and we will see how results concerning implications and equivalences between conditional orderings can be translated in terms of dependence notions.

Then we will point out some direct applications of our results to dependence notions related with conditions of default contagion and to the case of conditional independence between $X$ and $Y$.

More in details, the article is organized as follows. In Section 2 we first introduce some formal concepts needed to give a general definition of reversion of a conditional ordering. Then (Theorem 1) we point out a specific property, related with conditioning, of the $\leq_{\text {st }}$ order. Definitions and results given in Section 2 will be directly applied in Section 3, where we detail the specific conditional orderings of our interest and present Theorem 2. In a few words we show how each conditional ordering of the form (1) is equivalent 
to one of the form

$$
\mathcal{L}(X \mid Y \in J) \leq_{\widetilde{*}} \mathcal{L}\left(X \mid Y \in J^{\prime}\right),
$$

for suitable choice of the stochastic order $\leq_{\widetilde{*}}$ and of the intervals $J, J^{\prime}$.

The equivalence of a conditional ordering of the form (1) or of the form (2) with a corresponding concept of dependence will be treated in Section 4. Arguments presented therein will directly suggest the definition of a new positive dependence concept, that is related with the notion of Stochastic Increasing and that we denote by SIRL. Section 5 presents two different types of applications of Theorem 2: concepts of default contagion and cases of conditional independence between $X$ and $Y$. The latter application adds some potentially useful insight about positive dependence of conditionally independent random variables that are stochastically increasing w.r.t. a conditioning variable $Z$. Finally, we present a short discussion with some concluding remarks in Section 6. In the Appendix, we recall some notation and basic facts about stochastic dependence and copulas (see also $[8,13]$ ).

The choice of restricting our analysis to pairs of scalar random variables, besides allowing us to simplify notation and definitions, is also motivated by specially relevant symmetries related with reversing conditional orderings. Our arguments, however, admit suitable generalizations to the multivariate case.

\section{The role of usual stochastic ordering in con- ditioning}

Let $X, Y$ be two real-valued random variables. Let furthermore $E, E^{\prime}$ be events; $\mathcal{L}(X), \mathcal{L}(X \mid E), \mathcal{L}(Y), \mathcal{L}\left(Y \mid E^{\prime}\right)$ will denote respectively the probability laws of $X, X$ conditional on $E, Y, Y$ conditional on $E^{\prime}$. As a first issue in this section, we give a suitably general definition of stochastic monotonicity, of $Y$ w.r.t. $X$. To this purpose, it is convenient to define an order on the class $\mathcal{I}$ of all the intervals of $\mathbb{R}_{+}$.

Definition 1. For two intervals $I, I^{\prime}$ belonging to $\mathcal{I}$, we set $I \Subset I^{\prime}$ if $\inf I<$ $\inf I^{\prime}$ or $\inf I=\inf I^{\prime}, \sup I^{\prime}<\sup I$.

The relation $\Subset$ is symmetric, anti-reflexive and transitive and it defines a total order on $\mathcal{I}$.

Let $\leq_{*}$ denote a univariate stochastic order and let $\mathcal{A}, \mathcal{B}$ be two subclasses of $\mathcal{I}$. On the basis of Definition 1, we can now give the following definition. 
Definition 2. $Y$ is $\left(\leq_{*}, \mathcal{A}, \mathcal{B}\right)$-stochastically increasing in $X$ or stochastically increasing w.r.t. $X$ in the sense $\left(\leq_{*}, \mathcal{A}, \mathcal{B}\right)$ if and only if, for any $I \in \mathcal{A}, I^{\prime} \in \mathcal{B}, I \Subset I^{\prime}$

$$
\mathcal{L}(Y \mid X \in I) \leq_{*} \mathcal{L}\left(Y \mid X \in I^{\prime}\right)
$$

More shortly, we will also write $Y \uparrow_{\left(\leq_{*}, \mathcal{A}, \mathcal{B}\right)} X$.

Remark 1. By Definition 2, we obtain the classical notion of $Y$ stochastically increasing in $X(\mathrm{SI}(Y \mid X)$, see, e.g., the Appendix Section) by setting

$$
\text { * = st, } \mathcal{A}=\mathcal{B}=\{(x-\varepsilon, x+\varepsilon) \mid \varepsilon>0, x>\varepsilon\} .
$$

Definition 3. Let $\mathcal{A}, \mathcal{B}, \widetilde{\mathcal{A}}, \widetilde{\mathcal{B}}$ be classes of intervals and $*, \tilde{*}$ be stochastic orderings. The relation $Y \uparrow_{\left(\leq_{*}, \mathcal{A}, \mathcal{B}\right)} X$ is reverted by $X \uparrow_{\left(\leq_{\tilde{*}}, \widetilde{\mathcal{A}}, \widetilde{\mathcal{B}}\right)} Y$ if and only if, for any $I \in \mathcal{A}, I^{\prime} \in \mathcal{B}, J \in \widetilde{\mathcal{A}}, J^{\prime} \in \widetilde{\mathcal{B}}$,

$$
\mathcal{L}(Y \mid X \in I) \leq_{*} \mathcal{L}\left(Y \mid X \in I^{\prime}\right) \Leftrightarrow \mathcal{L}(X \mid Y \in J) \leq_{\tilde{*}} \mathcal{L}\left(X \mid Y \in J^{\prime}\right) .
$$

In this paper, we consider the special cases of Definition 2 obtained by combining the following choices:

1. $*=\mathrm{st}$,

2. $*=\mathrm{hr}$,

3. $*=\operatorname{lr}$

and
A. $\mathcal{L}(Y) \leq_{*} \mathcal{L}(Y \mid X>x) \quad \forall x>0$
B. $\mathcal{L}(Y \mid X>x) \leq_{*} \mathcal{L}\left(Y \mid X>x^{\prime}\right) \quad \forall x<x^{\prime}$,
C. $\mathcal{L}(Y \mid X=x) \leq_{*} \mathcal{L}\left(Y \mid X=x^{\prime}\right) \quad \forall x<x^{\prime}$.

By using the notation of Definition 2, we respectively have
A. $\mathcal{A}=\left\{\mathbb{R}_{+}\right\}$and $\mathcal{B}=\{(x,+\infty) \mid x>0\}$,
B. $\mathcal{A}=\mathcal{B}=\{(x,+\infty) \mid x>0\}$,
C. $\mathcal{A}=\mathcal{B}=\{(x-\varepsilon, x+\varepsilon) \mid \varepsilon>0, x>\varepsilon\}$.

The following result points out a property of the usual stochastic order that is relevant in our setting. This result will allow us to find triples $\left(\leq_{*}, \mathcal{A}, \mathcal{B}\right),\left(\leq_{\tilde{*}}, \widetilde{\mathcal{A}}, \widetilde{\mathcal{B}}\right)$ satisfying Definition 3 . 
Theorem 1. Let $A, B, B^{\prime}$ be intervals, with $B \Subset B^{\prime}$. Then intervals $A^{\prime}, D, D^{\prime}$ exist, with $D \Subset D^{\prime}$ and such that

$$
\mathcal{L}(Y \mid Y \in A, X \in B) \leq_{\text {st }} \mathcal{L}\left(Y \mid Y \in A, X \in B^{\prime}\right)
$$

is equivalent to

$$
\mathcal{L}\left(X \mid X \in A^{\prime}, Y \in D\right) \leq_{\text {st }} \mathcal{L}\left(X \mid X \in A^{\prime}, Y \in D^{\prime}\right) .
$$

Proof. By definition of usual stochastic order, inequalities (3), (4) also read as

$$
\begin{array}{r}
\mathrm{P}\{Y>y \mid Y \in A, X \in B\} \leq \mathrm{P}\left\{Y>y \mid Y \in A, X \in B^{\prime}\right\} \quad \forall y \geq 0, \\
\mathrm{P}\left\{X>x \mid X \in A^{\prime}, Y \in D\right\} \leq \mathrm{P}\left\{X>x \mid X \in A^{\prime}, Y \in D^{\prime}\right\} \quad \forall x \geq 0 .
\end{array}
$$

By Bayes' Theorem, Eq. (6) can be rewritten as

$$
\frac{\mathrm{P}\left\{X>x, X \in A^{\prime}, Y \in D\right\}}{\mathrm{P}\left\{X \in A^{\prime}, Y \in D\right\}} \leq \frac{\mathrm{P}\left\{X>x, X \in A^{\prime}, Y \in D^{\prime}\right\}}{\mathrm{P}\left\{X \in A^{\prime}, Y \in D^{\prime}\right\}}
$$

and subsequently

$$
\frac{\mathrm{P}\left\{Y \in D \mid X>x, X \in A^{\prime}\right\}}{\mathrm{P}\left\{Y \in D \mid X \in A^{\prime}\right\}} \leq \frac{\mathrm{P}\left\{Y \in D^{\prime} \mid X>x, X \in A^{\prime}\right\}}{\mathrm{P}\left\{Y \in D^{\prime} \mid X \in A^{\prime}\right\}} .
$$

On its turn, Eq. (5) becomes

$$
\frac{\mathrm{P}\{Y>y, Y \in A \mid X \in B\}}{\mathrm{P}\{Y \in A \mid X \in B\}} \leq \frac{\mathrm{P}\left\{Y>y, Y \in A \mid X \in B^{\prime}\right\}}{\mathrm{P}\left\{Y \in A \mid X \in B^{\prime}\right\}} .
$$

Thus, Eq.'s (5) and (6) are equivalent if and only if, for some $x, y>0$,

$$
\left\{\begin{array}{l}
D=A \\
D^{\prime}=A \cap(y,+\infty) \\
A^{\prime}=B \\
B^{\prime}=A^{\prime} \cap(x,+\infty) .
\end{array}\right.
$$

Since $A$ is given, $D \Subset D^{\prime}$. In fact, $\inf D^{\prime}=\max (y, \inf A) \geq \inf A$. We notice that, since intervals are connected sets, inf $I=\inf I^{\prime}$ implies $I \subset I^{\prime}$ or $I^{\prime} \subset I$. Hence the condition $\inf I=\inf I^{\prime}$ implies $I \cup I^{\prime} \Subset I \cap I^{\prime}$; therefore

- if $y>\inf A, A \Subset A \cap(y,+\infty)$;

- if $y \leq \inf A, A \cup(A \cap(y,+\infty)) \Subset A \cap(y,+\infty)$, that is, again, $A \Subset A \cap(y,+\infty)$. 
Remark 2. Theorem 1 implicitly provides a condition for the existence of the intervals $A^{\prime}, D, D^{\prime}$ and therefore for a conditional order being revertible. We notice that Eq. (7) is a system of four equations in the three unknowns $A^{\prime}, D, D^{\prime}$. The last equation allows us to check the existence of solutions for the system. Non-existence of solutions means that the considered conditional order is not revertible. When the solution exists, but at least one of the intervals $A^{\prime}, D, D^{\prime}$ is empty, then the considered conditional order is not revertible either.

Let $A, A^{\prime}, B, D$ be intervals as in Theorem 1 .

Proposition 1. $A \Subset A^{\prime}$ if and only if $D \Subset B$.

Proof. The claim straightly follows by the definition, given in the proof of Theorem 1, of the sets $A^{\prime}$ and $D$ in terms of $A$ and $B$.

Theorem 1 will have a basic role for our purposes in the next section. In this respect, it is useful to recall that the stochastic orderings $\leq_{\mathrm{hr}}, \leq_{\mathrm{lr}}$ can be characterized in terms of $\leq_{\text {st }}$ (see [17]). More precisely, one has

Proposition 2. $\mathcal{L}(S) \leq_{\mathrm{hr}} \mathcal{L}(T)$ if and only if, for any $t \geq 0$,

$$
\mathcal{L}(S-t \mid S>t) \leq_{\text {st }} \mathcal{L}(T-t \mid T>t) .
$$

Proposition 3. $\mathcal{L}(S) \leq_{\operatorname{lr}} \mathcal{L}(T)$ if and only if $\mathcal{L}(S \mid S \in A) \leq_{\text {st }} \mathcal{L}(T \mid T \in A)$ for any measurable set $A$.

In Definition 3, the interchange in the role of the variables $X, Y$ corresponds to a change in the stochastic order and in the choice of the conditioning events. We notice that there is a balance between the strength of the stochastic order and the strength of the conditioning events. Actually, in view of Propositions 2, 3, Proposition 1 guarantees that, if $\tilde{*}$ is stronger than $*$, then $D \Subset B$ and $D^{\prime} \Subset B^{\prime}$.

\section{Remarkable properties of conditional or- derings and related inversions}

In this section we analyze in details the conditional orderings defined by the positions 1., 2., 3., A., B., C., mentioned in Section 2. In particular by applying Theorem 1, we will find, for each entry, the corresponding reversed ordering. 
By combining A., B., C. with cases 1., 2., 3., we obtain the following matrices $M(Y \mid X), M(X \mid Y)$ :

\begin{tabular}{c|c|c|c|} 
& $\mathrm{A}$ & $\mathrm{B}$ & $\mathrm{C}$ \\
\hline 1 & $\mathrm{~A} 1(Y \mid X)$ & $\mathrm{B} 1(Y \mid X)$ & $\mathrm{C} 1(Y \mid X)$ \\
\hline 2 & $\mathrm{~A} 2(Y \mid X)$ & $\mathrm{B} 2(Y \mid X)$ & $\mathrm{C} 2(Y \mid X)$ \\
\hline 3 & $\mathrm{~A} 3(Y \mid X)$ & $\mathrm{B} 3(Y \mid X)$ & $\mathrm{C} 3(Y \mid X)$ \\
\hline
\end{tabular}

\begin{tabular}{c|c|c|c|} 
& $\mathrm{A}$ & $\mathrm{B}$ & $\mathrm{C}$ \\
\hline 1 & $\mathrm{~A} 1(X \mid Y)$ & $\mathrm{B} 1(X \mid Y)$ & $\mathrm{C} 1(X \mid Y)$ \\
\hline 2 & $\mathrm{~A} 2(X \mid Y)$ & $\mathrm{B} 2(X \mid Y)$ & $\mathrm{C} 2(X \mid Y)$ \\
\hline 3 & $\mathrm{~A} 3(X \mid Y)$ & $\mathrm{B} 3(X \mid Y)$ & $\mathrm{C} 3(X \mid Y)$ \\
\hline
\end{tabular}

Each entry of the two matrices $M(Y \mid X), M(X \mid Y)$ is a property of conditional order. For example, $\mathrm{A} 1(Y \mid X)$ means $\mathcal{L}(Y) \leq_{s t} \mathcal{L}(Y \mid X>x) \quad \forall x>0$.

Remark 3. Heuristically speaking, the conditional orderings appearing in the matrices become stronger and stronger when reading their entries "from above to below" or "from left to right". In view of the chain of implications

$$
\leq_{\mathrm{lr}} \Rightarrow \leq_{\mathrm{hr}} \Rightarrow \leq_{\mathrm{st}},
$$

we immediately obtain $A 3 \Rightarrow A 2 \Rightarrow A 1, B 3 \Rightarrow B 2 \Rightarrow B 1, C 3 \Rightarrow C 2 \Rightarrow C 1$. On the other hand, the implications $C 1 \Rightarrow B 1 \Rightarrow A 1, C 2 \Rightarrow B 2 \Rightarrow A 2$, $C 3 \Rightarrow B 3 \Rightarrow A 3$ follow from the relation $\mathbb{R}_{+} \Subset(x,+\infty) \Subset\{x\}$ in view of Theorem 1 and Proposition 1.

In principle the two matrices $M(Y \mid X), M(X \mid Y)$ present $18=9+9$ different properties for the joint law of $(X, Y)$. Based on the existing literature (see e.g. $[17,7,19]$ ), we can guess however the existence of some equivalences among them. Actually a complete catalogue of equivalences can be established between pairs of them in terms of Definition 3 and Theorem 1. More precisely, we have the following result.

Theorem 2. The matrix $M(X \mid Y)$ is the transpose of $M(Y \mid X)$.

Proof. We apply Theorem 1 to any property corresponding to the entries of $M(Y \mid X)$. We provide the detailed proof for the elements in the first column of $M(Y \mid X)$ (thus obtaining the first row of $M(X \mid Y)$ ). The other equivalences can be proven by analogous arguments. 
A1 $(Y \mid X)$ satisfies condition (3) with $A=B=\mathbb{R}_{+}, B^{\prime}=\left(x^{\prime},+\infty\right)$, $\forall x^{\prime}>0$. Eq. (7) in the proof of Theorem 1 allows us to write the equivalent condition (4) with $A^{\prime}=D=\mathbb{R}_{+}, D^{\prime}=(y,+\infty), \forall y>0$. Finally, we see that $B^{\prime}=\left(x^{\prime},+\infty\right)$ satisfies the last condition of the system $(7)$, $B^{\prime}=A^{\prime} \cap(x,+\infty)$. Hence, by Theorem $1, \mathrm{~A} 1(Y \mid X)$ is equivalent to the inequality (4), that turns out to be equivalent to $\mathrm{A} 1(X \mid Y)$.

In view of Proposition 2, $\mathrm{A} 2(Y \mid X)$ is equivalent to condition (3) with $A=(t,+\infty), \forall t \geq 0 ; B=\mathbb{R}_{+}, B^{\prime}=\left(x^{\prime},+\infty\right), \forall x^{\prime}>0$. By Theorem 1 , $\mathrm{A} 2(Y \mid X)$ is equivalent to (4) with $A^{\prime}=\mathbb{R}_{+}, D=(t,+\infty), \forall t \geq 0$;

$D^{\prime}=(\max (t, y),+\infty), \forall y>0$. Since $\max (t, y) \geq y$, (4) turns out to be equivalent to $\mathrm{B} 1(X \mid Y)$.

In view of Proposition 3, A3 $(Y \mid X)$ is equivalent to condition (3) with $A=(t-\varepsilon, t+\varepsilon), \forall \varepsilon>0, t>\varepsilon ; B=\mathbb{R}_{+}, B^{\prime}=\left(x^{\prime},+\infty\right), \forall x^{\prime}>0$. By Theorem $1, \mathrm{~A} 3(Y \mid X)$ is equivalent to (4) with $A^{\prime}=\mathbb{R}_{+}$,

$D=(t-\varepsilon, t+\varepsilon), \forall \varepsilon>0, t>\varepsilon ; D^{\prime}=(t-\varepsilon, t+\varepsilon) \cap(y,+\infty), \forall y>0$. $D^{\prime}=(\max (y, t-\varepsilon), t+\varepsilon) \subset D$ and therefore $D \Subset D^{\prime}$ also holds. In other words, in the limit for $\varepsilon$ going to $0, D^{\prime}$ collapses in a point on the right of $\{t\}$. Therefore (4) turns out to be equivalent to $\mathrm{C} 1(X \mid Y)$.

In view of Theorem 2 , the table in (8) also reads

\begin{tabular}{|l|l|l|}
\hline $\mathrm{A} 1(Y \mid X)$ & $\mathrm{B} 1(Y \mid X)$ & $\mathrm{C} 1(Y \mid X)$ \\
\hline $\mathrm{B} 1(X \mid Y)$ & $\mathrm{A} 2(Y \mid X)$ & $\mathrm{C} 2(Y \mid X)$ \\
\hline $\mathrm{C} 1(X \mid Y)$ & $\mathrm{C} 2(X \mid Y)$ & $\mathrm{C} 3(Y \mid X)$ \\
\hline
\end{tabular}

Remark 4. 1. The hazard rate order $\leq_{\mathrm{hr}}$ can be characterized as follows (see [17]): $Y \geq_{\mathrm{hr}} X$ if and only if

$$
\frac{\bar{G}_{X}(t)}{\bar{G}_{Y}(t)} \text { is decreasing in } t \text {. }
$$

Eq. (12) allows us to define $\leq_{\mathrm{hr}}$ even if one or both the distributions to be compared are not absolutely continuous.

2. For what concerns the likelihood ratio order, a characterization not involving densities can be given as follows: $X \leq_{\operatorname{lr}} Y$ if and only if

$$
P\{X \in A\} P\{Y \in B\} \geq P\{X \in B\} P\{Y \in A\}
$$

for all measurable sets $A$ and $B$ such that $A \leq B$, where $A \leq B$ means that $x \in A$ and $y \in B$ imply that $x \leq y$. 
All our results and the conditional orderings considered so far do not require absolute continuity.

In the absolutely continuous case, the proof of some of the equivalences in Theorem 2 could also have been directly obtained by applying Bayes formula. In particular, we can for instance argue as follows:

- $\mathrm{C} 2(Y \mid X) \Leftrightarrow \mathcal{L}(Y \mid X=x) \leq_{\mathrm{hr}} \mathcal{L}\left(Y \mid X=x^{\prime}\right) \forall x<x^{\prime} \Leftrightarrow$

$$
\begin{gathered}
\frac{\bar{G}_{Y}\left(y^{\prime} \mid X=x\right)}{\bar{G}_{Y}(y \mid X=x)} \leq \frac{\bar{G}_{Y}\left(y^{\prime} \mid X=x^{\prime}\right)}{\bar{G}_{Y}\left(y \mid X=x^{\prime}\right)} \forall x<x^{\prime}, y<y^{\prime} \Leftrightarrow \\
\frac{g_{X}\left(x^{\prime} \mid Y>y\right)}{g_{X}(x \mid Y>y)} \leq \frac{g_{X}\left(x^{\prime} \mid Y>y^{\prime}\right)}{g_{X}\left(x \mid Y>y^{\prime}\right)} \forall x<x^{\prime}, y<y^{\prime} \Leftrightarrow \\
\mathcal{L}(X \mid Y>y) \leq_{\operatorname{lr}} \mathcal{L}\left(X \mid Y>y^{\prime}\right) \forall y<y^{\prime} \Leftrightarrow \mathrm{B} 3(X \mid Y) ; \\
\text { • } \mathrm{A} 3(Y \mid X) \Leftrightarrow \mathcal{L}(Y) \leq \operatorname{lr} \mathcal{L}(Y \mid X>x) \Leftrightarrow \\
\bar{G}_{X}(x \mid Y=y) \leq \bar{G}_{X}\left(x \mid Y=y^{\prime}\right) \forall y<y^{\prime} \Leftrightarrow \\
\mathcal{L}(X \mid Y=y) \leq{ }_{\mathrm{st}} \mathcal{L}\left(X \mid Y=y^{\prime}\right) \Leftrightarrow \mathrm{C} 1(X \mid Y) ; \\
\left.\bar{G}_{Y}(Y \mid X) \Leftrightarrow \mathcal{L}(Y \mid X>x) \leq y_{\mathrm{hr}} \mathcal{L}^{\prime}|X| X>x^{\prime}\right) \forall x<x^{\prime} \Leftrightarrow \bar{G}_{Y}(y \mid X>x) \geq \bar{G}_{Y}\left(y \mid X>x^{\prime}\right) \bar{G}_{Y}\left(y^{\prime} \mid X>x\right) \forall x<x^{\prime}, y<y^{\prime} \Leftrightarrow \\
\bar{G}_{X}\left(x^{\prime} \mid Y>y^{\prime}\right) \bar{G}_{X}(x \mid Y>y) \geq \bar{G}_{X}\left(x \mid Y>y^{\prime}\right) \bar{G}_{X}\left(x^{\prime} \mid Y>y\right) \forall x<x^{\prime}, y<y^{\prime} \\
\Leftrightarrow \mathcal{L}(X \mid Y>y) \leq{ }_{\mathrm{hr}} \mathcal{L}\left(X \mid Y>y^{\prime}\right) \forall y<y^{\prime} \Leftrightarrow \mathrm{B} 2(X \mid Y) .
\end{gathered}
$$

Remark 5. The properties A1, B2, C3 lie on the main diagonal of the matrix $M(Y \mid X)$. Therefore, in view of Theorem 2, they must be symmetric w.r.t. $X, Y$, i.e. $A 1(Y \mid X)=A 1(X \mid Y), B 2(Y \mid X)=B 2(X \mid Y), C 3(Y \mid X)=$ $C 3(X \mid Y)$. In these cases, we notice that the interchange between $X, Y$ does not require a balancing between a change in the stochastic order and in the conditioning events.

Remark 6. When $X, Y$ are exchangeable, $M(Y \mid X)=M(X \mid Y)$. 


\section{Conditional orderings and dependence}

All the entries of the matrices $M(Y \mid X), M(X \mid Y)$ can be seen as dependence properties for the pair $(X, Y)$. In some cases the correspondences are well known or immediately follow by definitions. This is the case for A1, B1, C1, C3 (see the Appendix and the table in (14) below). This section will be devoted to analyzing the remaining ones.

The following result is a simple consequence of Bayes formula.

Proposition 4. $B 2(Y \mid X) \Leftrightarrow(X, Y)$ is RCSI.

Proof. By Eq. (12), B2(Y|X), i.e. $\mathcal{L}(Y \mid X>x) \leq_{\mathrm{hr}} \mathcal{L}\left(Y \mid X>x^{\prime}\right)$ for any $x<x^{\prime}, y<y^{\prime}$, can be rewritten as

$$
\bar{G}_{Y}\left(y^{\prime} \mid X>x^{\prime}\right) \bar{G}_{Y}(y \mid X>x) \geq \bar{G}_{Y}\left(y \mid X>x^{\prime}\right) \bar{G}_{Y}\left(y^{\prime} \mid X>x\right)
$$

for any $x<x^{\prime}, y<y^{\prime}$. By applying Bayes formula the inequality

$$
\bar{F}\left(x^{\prime}, y^{\prime}\right) \bar{F}(x, y) \geq \bar{F}\left(x, y^{\prime}\right) \bar{F}\left(x^{\prime}, y\right)
$$

is obtained, that is the definition of $\bar{F} T P_{2}$, i.e. $(X, Y)$ is RCSI (see the Appendix and [13]).

In view of the above arguments, we can rewrite $M(Y \mid X)$ in the form:

\begin{tabular}{|c|c|c|}
\hline PQD & $\operatorname{RTI}(Y \mid X)$ & $\mathrm{SI}(Y \mid X)$ \\
\hline $\operatorname{RTI}(X \mid Y)$ & RCSI & $\mathrm{C} 2(Y \mid X)$ \\
\hline $\mathrm{SI}(X \mid Y)$ & $\mathrm{C} 2(X \mid Y)$ & $\mathrm{PLRD}$ \\
\hline
\end{tabular}

On the main diagonals of $M(Y \mid X)$ and $M(X \mid Y)$, we find the symmetric dependence properties PQD, RCSI, PLRD.

We aim now at completing the correspondences between dependence properties and entries of $M(Y \mid X)$.

Also $\mathrm{C} 2(Y \mid X), \mathrm{C} 2(X \mid Y)$ can be interpreted as conditions of stochastic dependence. However they do not correspond, as far as we know, to any definition introduced so far in the literature. In this respect we state the following

Proposition 5. C2 $(Y \mid X)$ holds if and only if $Y-t \mid Y>t$ is SI in $X$ for any $t>0$. 
Proof. By Eq. (12), the condition C2 $(Y \mid X)$, i.e.

$$
\mathcal{L}(Y \mid X=x) \leq_{\text {hr }} \mathcal{L}\left(Y \mid X=x^{\prime}\right)
$$

is equivalent to

$$
\bar{G}_{Y}\left(y^{\prime} \mid X=x^{\prime}\right) \bar{G}_{Y}(y \mid X=x) \geq \bar{G}_{Y}\left(y \mid X=x^{\prime}\right) \bar{G}_{Y}\left(y^{\prime} \mid X=x\right)
$$

for any $x<x^{\prime}, y<y^{\prime}$, that is $\frac{\bar{G}_{Y}\left(y^{\prime} \mid X=x\right)}{\bar{G}_{Y}(y \mid X=x)}$ is increasing in $x$. Since $y<y^{\prime}$, we can write $y^{\prime}=y+t$, for $t>0$, and thus

$$
\frac{\bar{G}_{Y}(y+t \mid X=x)}{\bar{G}_{Y}(y \mid X=x)}=\bar{G}_{Y}(y+t \mid X=x, Y>y) \uparrow x \quad \forall t>0 .
$$

In view of the above result, it is natural to give the following

Definition 4. $Y$ is Stochastically Increasing in $X$ in the Residual Lifetime (in short $\operatorname{SIRL}(Y \mid X))$ if $\operatorname{SI}(Y-t \mid Y>t, X)$ for any $t>0$.

The matrix $M(Y \mid X)$ can be finally rewritten as

\begin{tabular}{|c|c|c|}
\hline PQD & $\operatorname{RTI}(Y \mid X)$ & $\operatorname{SI}(Y \mid X)$ \\
\hline $\operatorname{RTI}(X \mid Y)$ & $\operatorname{RCSI}$ & $\operatorname{SIRL}(Y \mid X)$ \\
\hline $\operatorname{SI}(X \mid Y)$ & $\operatorname{SIRL}(X \mid Y)$ & $\operatorname{PLRD}$ \\
\hline
\end{tabular}

Condition $\operatorname{SIRL}(Y \mid X)$ is a stronger dependence property than $\operatorname{SI}(Y \mid X)$. In particular, the chain of equivalences (10) implies: PLRD $\Rightarrow$ SIRL $\Rightarrow$ RCSI. Concerning the identification of SIRL with a property of stochastic dependence, it is of interest to note that the following result relates such a property with the behavior of the survival copula $\hat{C}(u, v)=\bar{F}\left(\bar{G}_{X}^{-1}(u), \bar{G}_{Y}^{-1}(v)\right)$ (see also the Appendix).

Proposition 6. $Y$ is SIRL in $X$ if and only if $\frac{\partial \hat{C}}{\partial u}(u, v)$ is $\mathrm{TP}_{2}$.

Proof. The term $\bar{G}_{Y}(y+t \mid X=x, Y>t)$ can also be rewritten as

$$
\frac{P\{X=x, Y>y+t\}}{P\{X=x, Y>t\}}=\frac{\frac{\partial \hat{C}}{\partial u}\left(\bar{G}_{X}(x), \bar{G}_{Y}(y+t)\right)}{\frac{\partial \hat{C}}{\partial u}\left(\bar{G}_{X}(x), \bar{G}_{Y}(t)\right)} .
$$


Since this function has to be increasing in $x$, by adopting the change of variables

$$
u=\bar{G}_{X}(x), u^{\prime}=\bar{G}_{X}\left(x^{\prime}\right), v=\bar{G}_{Y}(y+t), z=\bar{G}_{Y}(t),
$$

we obtain

$$
\frac{\partial \hat{C}}{\partial u}(u, v) \frac{\partial \hat{C}}{\partial u}\left(u^{\prime}, z\right) \leq \frac{\partial \hat{C}}{\partial u}\left(u^{\prime}, v\right) \frac{\partial \hat{C}}{\partial u}(u, z) .
$$

Since $x<x^{\prime}<t<y+t$ and, therefore, $u>u^{\prime}, z>v$, Eq. (17) corresponds to $\frac{\partial \hat{C}}{\partial u}(u, v)$ being $\mathrm{TP}_{2}$.

In view of Proposition 6 and Definition 8 in the Appendix, the conditional orderings treated here can be written as properties of a copula. This circumstance is not surprising, since conditional orderings are not affected by strictly increasing (deterministic) transformations, as it happens for copulas.

If the copula is symmetric, in particular in the exchangeable case, $M(Y \mid X)=M(X \mid Y)$, therefore we have in particular (for both the matrices)

$$
\begin{aligned}
\mathrm{B} 1 & \Leftrightarrow \mathrm{A} 2 \\
\mathrm{~B} 3 & \Leftrightarrow \mathrm{C} 2 \\
\mathrm{C} 1 & \Leftrightarrow \mathrm{A} 3
\end{aligned}
$$

In the case when the copula is Archimedean, we have the further equivalences:

$$
\begin{aligned}
& \mathrm{B} 1 \Leftrightarrow \mathrm{B} 2 \text { (see e.g. [3]) } \\
& \mathrm{C} 1 \Leftrightarrow \mathrm{C} 2
\end{aligned}
$$

as stated by the following proposition.

Proposition 7. Let $\hat{C}$ be an Archimedean copula. Then SI $\Rightarrow$ SIRL.

Proof. Let be $\hat{C}(u, v)=\phi\left(\phi^{-1}(u)+\phi^{-1}(v)\right)$. $\hat{C}$ is SI if and only if, for any $a, x \geq 0, \frac{\phi^{\prime}(x+a)}{\phi^{\prime}(x)}$ is increasing w.r.t. $x$, i.e., for any $x<x^{\prime}$,

$$
\frac{\phi^{\prime}(x+a)}{\phi^{\prime}(x)} \leq \frac{\phi^{\prime}\left(x^{\prime}+a\right)}{\phi^{\prime}\left(x^{\prime}\right)} .
$$

In view of Proposition 6, the thesis consists in $\frac{\partial \hat{C}}{\partial u}(u, v)$ being $T P_{2}$, i.e., for any $u, u^{\prime}, v, v^{\prime} \in[0,1], u<u^{\prime}, v<v^{\prime}$,

$$
\phi^{\prime}\left(\alpha^{\prime}+\beta\right) \phi^{\prime}\left(\alpha+\beta^{\prime}\right) \leq \phi^{\prime}(\alpha+\beta) \phi^{\prime}\left(\alpha^{\prime}+\beta^{\prime}\right) \text {, }
$$

where $\alpha=\phi^{-1}(u), \alpha^{\prime}=\phi^{-1}\left(u^{\prime}\right), \beta=\phi^{-1}(v), \beta^{\prime}=\phi^{-1}\left(v^{\prime}\right)$ and therefore $\alpha^{\prime}<\alpha, \beta^{\prime}<\beta$. By choosing $x=\alpha^{\prime}+\beta^{\prime}, x^{\prime}=\alpha+\beta^{\prime}, a=\beta-\beta^{\prime}$, we obtain that Eq. (18) implies Eq. (19). 


\section{Applications of Theorem 2}

The circumstance that each entry of the matrix $M(Y \mid X)$ is equivalent to an entry of $M(X \mid Y)$ turns out to be of interest in several different contexts. Some of them will be sketched in what follows.

\subsection{Default contagion and dynamic dependence prop- erties}

In several applied models, the following type of conditional ordering can be of interest:

$$
\mathcal{L}(Y \mid X=x) \leq_{*} \mathcal{L}(Y \mid X>x)
$$

It is natural to wonder whether property (20) is different from those appearing in $M(Y \mid X)$. Actually, such stochastic inequalities are equivalent to the ones appearing in the column B. of $M(Y \mid X)$. More precisely we can state

Proposition 8. For $*=\mathrm{st}$, hr, lr, condition (20) is equivalent to

$$
\mathcal{L}(Y \mid X>x) \leq_{*} \mathcal{L}\left(Y \mid X>x^{\prime}\right), \forall x<x^{\prime}
$$

Proof.

$$
\begin{aligned}
& \text { - } \mathcal{L}(Y \mid X=x) \leq_{\text {st }} \mathcal{L}(Y \mid X>x) \Leftrightarrow \\
& \frac{\bar{G}_{X}(x)}{g_{X}(x)} \leq \frac{\bar{G}_{X}(x \mid Y>y)}{g_{X}(x \mid Y>y)} \Leftrightarrow \mathcal{L}(X) \leq_{\mathrm{hr}} \mathcal{L}(X \mid Y>y),
\end{aligned}
$$

that, in view of Theorem 2, is equivalent to $\mathrm{B} 1(Y \mid X)$.

- $\mathcal{L}(Y \mid X=x) \leq_{\mathrm{hr}} \mathcal{L}(Y \mid X>x)$

$$
\begin{gathered}
\Leftrightarrow \frac{\bar{G}_{Y}\left(y^{\prime} \mid X=x\right)}{\bar{G}_{Y}(y \mid X=x)} \leq \frac{\bar{G}_{Y}\left(y^{\prime} \mid X>x\right)}{\left.\bar{G}_{Y}(y \mid X>x)\right)}, \forall y<y^{\prime} \Leftrightarrow \\
\frac{\bar{G}_{X}(x \mid Y>y)}{g_{X}(x \mid Y>y)} \leq \frac{\bar{G}_{X}\left(x \mid Y>y^{\prime}\right)}{g_{X}\left(x \mid Y>y^{\prime}\right)}, \forall y<y^{\prime} \Leftrightarrow
\end{gathered}
$$

$\mathcal{L}(X \mid Y>y) \leq_{\text {hr }} \mathcal{L}\left(X \mid Y>y^{\prime}\right)$, that is $\mathrm{B} 2(Y \mid X)$.

- $\mathcal{L}(Y \mid X=x) \leq_{\operatorname{lr}} \mathcal{L}(Y \mid X>x) \Leftrightarrow \frac{\bar{G}_{X}(x \mid Y=y)}{g_{X}(x \mid Y=y)} \leq \frac{\bar{G}_{X}\left(x \mid Y=y^{\prime}\right)}{g_{X}\left(x \mid Y=y^{\prime}\right)}$

$\Leftrightarrow \mathcal{L}(X \mid Y=y) \leq_{\mathrm{hr}} \mathcal{L}\left(X \mid Y=y^{\prime}\right)$, i.e. B3 $(Y \mid X)$. 
We notice that all the three parts of the above proof are based on Bayes formula and on Theorem 2 .

In different frameworks, especially in the case when $X, Y$ are non-negative random variables with the meaning of failure times, default times or times to events, it is important to establish whether the following condition holds:

$$
\mathcal{L}(Y \mid X=t, Y>t) \leq_{*} \mathcal{L}(Y \mid X>t, Y>t)
$$

The stochastic inequality (21) is a special case of dependence properties of dynamic type studied in the field of reliability; see in particular $[1,2,14,16]$. The same inequality is also closely related to the concept of default contagion introduced in the literature on financial risk; see e.g. [12] and references therein. Some relations between the inequalities in (20) and in (21) are made precise by the following result.

Proposition 9. For $*=\mathrm{hr}$, lr, condition (20) implies (21).

Proof. $\quad$ - For $*=\mathrm{lr}$, the implication straightly follows by

[17, Theorem 1.C.6].

- For $*=\mathrm{hr}$, it is a consequence of the implication:

$$
\mathcal{L}(S) \leq_{\mathrm{hr}} \mathcal{L}(T) \Rightarrow \mathcal{L}(S \mid S>t) \leq_{\mathrm{hr}} \mathcal{L}(T \mid T>t), \forall t \geq 0 .
$$

Propositions 8, 9, applied to the results of the previous section, lead us to link dependence properties to default contagion properties.

Corollary 1. $\quad \operatorname{SIRL}(X \mid Y) \Rightarrow$

$$
\mathcal{L}(Y \mid X=t, Y>t) \leq_{\operatorname{lr}} \mathcal{L}(Y \mid X>t, Y>t)
$$

- $\operatorname{SIRL}(Y \mid X) \Rightarrow \mathcal{L}(X \mid X>t, Y=t) \leq_{\operatorname{lr}} \mathcal{L}(X \mid X>t, Y>t)$

- $(X, Y) R C S I \Rightarrow \mathcal{L}(Y \mid X=t, Y>t) \leq_{\mathrm{hr}} \mathcal{L}(Y \mid X>t, Y>t)$ and

$$
\mathcal{L}(X \mid X>t, Y=t) \leq_{\text {hr }} \mathcal{L}(X \mid X>t, Y>t) .
$$

Remark 7. $(X, Y)$ RCSI also implies default contagion in the usual stochastic order sense, i.e.

$$
\mathcal{L}(Y \mid X=t, Y>t) \leq_{\text {st }} \mathcal{L}(Y \mid X>t, Y>t)
$$

and

$$
\mathcal{L}(X \mid X>t, Y=t) \leq_{\text {st }} \mathcal{L}(X \mid X>t, Y>t) .
$$




\subsection{Conditional independence and reversed Markov pro- cesses}

Let $X, Y$ be conditionally independent w.r.t. a random variable $Z$. It is well known that, when $X$ and $Y$ are both stochastically increasing w.r.t. $Z$, in some suitable sense, then the pair $(X, Y)$ manifests some corresponding property of positive dependence. A rich literature has been devoted to this issue, for the general case of $n \geq 2$ conditionally independent variables. See, in particular, $[9,10,11,18,20]$.

In this subsection, we show some specific aspects of this topic for the case of the bivariate dependence notions appearing in the matrices $M(Y \mid X), M(Y \mid X)$.

The following definition of transitivity of a dependence property is relevant in the present setting.

Definition 5. Let $\mathcal{D}$ be a dependence property. We say that $\mathcal{D}$ is transitive under conditional independence, in short c.i.-transitive, if the conditions:

i) $(X, Z)$ satisfies $\mathcal{D}$,

ii) $(Z, Y)$ satisfies $\mathcal{D}$,

iii) $X, Y$ conditionally independent given $Z$

imply that $(X, Y)$ satisfies $\mathcal{D}$.

For example, the following implication is clear (under the assumption that $X, Y$ are conditionally independent given $Z$ ): if

$$
\mathcal{L}\left(Z \mid X=x^{\prime}\right) \leq_{\text {st }} \mathcal{L}\left(Z \mid X=x^{\prime \prime}\right) \text { for any } x^{\prime}<x^{\prime \prime}
$$

and

$$
\mathcal{L}\left(Y \mid Z=z^{\prime}\right) \leq_{\text {st }} \mathcal{L}\left(Y \mid Z=z^{\prime \prime}\right) \text { for any } z^{\prime}<z^{\prime \prime}
$$

then

$$
\mathcal{L}\left(Y \mid X=x^{\prime}\right) \leq_{\text {st }} \mathcal{L}\left(Y \mid X=x^{\prime \prime}\right) \text { for any } x^{\prime}<x^{\prime \prime} ;
$$

in other words $C 1(Z \mid X)$ and $C 1(Y \mid X)$ imply $C 1(Y \mid Z)$, i.e. SI is c.i.-transitive.

The notions of PQD, RTI and PLRD are also c.i.-transitive. It is also obvious, by definition of c.i.-transitivity and by Theorem 2 , that a property $\mathcal{D}$ is c.i.-transitive if and only if its reversed property $\mathcal{D}^{*}$ is such.

Proposition 10. Let $X, Y$ be conditionally independent given $Z$. Then the following implications hold:

a) if $(X, Z)$ and $(Y, Z)$ are $\mathrm{PQD}$, then $(X, Y)$ is $\mathrm{PQD}$; 
b) if $(X, Z)$ and $(Y, Z)$ are PLRD, then $(X, Y)$ is PLRD;

c) if $\mathrm{A} 3(X \mid Z)$ and $\operatorname{SI}(Y \mid Z)$ hold, then $\operatorname{SI}(Y \mid X)$;

d) if $\mathrm{B} 3(X \mid Z)$ and $\operatorname{SIRL}(Y \mid Z)$ hold, then $\operatorname{SIRL}(Y \mid X)$.

Proof. The proof of a) and b) is immediate, by taking into account Definition 5 and the fact that PQD and PLRD are symmetric. The proof of c) is also almost obvious. In fact, by Theorem $2, \mathrm{~A} 3(X \mid Z)$ is equivalent to $\mathrm{C} 1(Z \mid X)$ i.e. $\operatorname{SI}(X \mid Z)$. Then $\mathrm{C} 1(Y \mid X)$ follows by c.i.-transitivity of $C 1$. Similarly for item d).

Items c) and d) of Proposition 10 are slightly different from other results given in the literature cited above. In fact, in such results, one considers random variables $T_{1}, \ldots, T_{n}$ that are conditionally independent given $Z$ and it is typically assumed that one and the same conditional ordering holds for all the (possibly different) conditional distributions of $T_{j}$ given $Z$. Compare in particular item c) with [9] or [20, p. 138].

Interest of c.i.-transitive dependence properties also arises in a natural way in the analysis of real-valued Markov processes. Consider a Markov process in discrete time, $X_{0}, X_{1}, \ldots$, with transition kernel $p\left(x \mid x^{\prime}\right)$, and, for $n=2,3, \ldots$, let $p^{(n)}\left(x \mid x^{\prime}\right)$ be the transition kernel in $n$ steps.

Lemma 1. Let $p\left(x \mid x^{\prime}\right)$ satisfy a dependence property $\mathcal{D}$. If $\mathcal{D}$ is transitive, then also $p^{(n)}\left(x \mid x^{\prime}\right)$ satisfies $\mathcal{D}$, for $n=2,3, \ldots$.

Proof. $p^{(2)}\left(x \mid x^{\prime}\right)$ satisfies $\mathcal{D}$ just by Definition 5 . For $n=3,4, \ldots$, the claim follows by induction. In fact $\left(X_{0}, X_{2}\right)$ satisfies $D,\left(X_{2}, X_{3}\right)$ satisfies $\mathcal{D}$, and $X_{0}, X_{3}$ are conditionally independent w.r.t. $X_{2}$ and so on.

We obtain the following easy result.

Proposition 11. Let $n=1,2, \ldots$

a) If $\left(X_{0}, X_{1}\right)$ is $\mathrm{PQD}$, then also $\left(X_{n}, X_{0}\right)$ is $\mathrm{PQD}$;

b) if $\left(X_{0}, X_{1}\right)$ is PLRD, then also $\left(X_{n}, X_{0}\right)$ is PLRD;

c) if $X_{1}$ is $\mathrm{SI}$ in $X_{0}$, then $\left(X_{n}, X_{0}\right)$ satisfies $\mathrm{A} 3\left(X_{0} \mid X_{n}\right)$;

d) if $\left(X_{0}, X_{1}\right)$ is $\operatorname{SIRL}\left(X_{1} \mid X_{0}\right)$, then $\left(X_{n}, X_{0}\right)$ is $\operatorname{B} 3\left(X_{0} \mid X_{n}\right)$. 


\section{Discussion and conclusions}

We conclude the article with some comments and final remarks.

Our main results are Theorem 1 and Theorem 2. These results present advantages both of theoretical and technical type. More precisely, Theorem 2 provides a unified framework for the proofs of the identity between the matrix $M(Y \mid X)$ and the transpose of the matrix $M(X \mid Y)$. Theorem 1 is a general result about the usual stochastic order that can be applied to Theorem 2, by using Propositions 2, 3. We notice that Theorem 1 does not require any regularity condition on the probability distributions of the involved variables. In particular it does not rely on absolute continuity, thus making Theorem 2 independent of this assumption as well.

The use of Theorem 1 in the proof of Theorem 2 is made possible by Propositions 2, 3, which allow us to express $\leq_{\mathrm{lr}}, \leq_{\mathrm{hr}}$ in terms of $\leq_{\mathrm{st}}$.

Theorem 1 could be similarly applied to conditional orderings involving other stochastic orders that are reducible to $\leq_{\text {st }}$. As a first instance, we can refer to the reversed hazard rate order, that can be characterized in terms of $\leq_{\text {st }}$ similarly to the hazard rate order. However also other stochastic orderings can be linked to $\leq_{\text {st }}$ by means of different characterizations: this is the case, for example, of the mean residual life order, the harmonic mean residual life order, the convex order, the dispersive order (see [17], in particular Theorems 2.A.4, 2.B.2, 3.A.4, 3.B.13).

Initially our work has been inspired by the purpose of comparing the univariate stochastic orderings $\leq_{\mathrm{st}}, \leq_{\mathrm{hr}}, \leq_{\mathrm{lr}}$ in terms of differences among notions of conditional orderings. In this respect, we actually pointed out some symmetries that exist between the notions of stochastic orders $\leq_{\text {st }}$, $\leq_{\mathrm{hr}}, \leq_{\mathrm{lr}}$ on one side and conditional orderings on the other side. Our results also provide some insight about the concepts of positive dependence. In particular, by means of Theorem 2, we can explain the superpositions between different pairs of concepts of positive dependence.

Theorems 1, 2 are also useful to understanding the link between notions of default contagion and other notions of positive dependence properties (see Corollary 1).

All our results can be easily extended to a particular case of multivariate conditioning, i.e. in the case when conditioning events differ each other by the specification of only one variable, as it happens for example between

$$
\begin{aligned}
& E_{0}=\left\{T_{1}=t_{1}, \ldots, T_{k}=t_{k}, T_{k+1}>0, T_{k+2}>0, T_{k+3}>s_{3}, \ldots, T_{n}>s_{n-k}\right\}, \\
& E=\left\{T_{1}=t_{1}, \ldots, T_{k}=t_{k}, T_{k+1}>s_{1}, T_{k+2}>0, T_{k+3}>s_{3}, \ldots, T_{n}>s_{n-k}\right\} .
\end{aligned}
$$

In view of transitivity of conditioning, we can read a comparison of the kind 


$$
\begin{aligned}
\mathcal{L}\left(T_{k+2} \mid E_{0}\right) \leq_{*} \mathcal{L}\left(T_{k+2} \mid E\right) \text { as } & \\
& \mathcal{L}\left(T_{k+2} \mid E_{0}\right) \leq_{*} \mathcal{L}\left(\left(T_{k+2} \mid E_{0}\right) \mid T_{k+1}>s_{1}\right)
\end{aligned}
$$

and trace it back to the bivariate case treated so far, with

$$
X=T_{k+1}\left|E_{0}, Y=T_{k+2}\right| E_{0} .
$$

Our results can be suitably extended from the bivariate to the multivariate case. Provided appropriate "weak" notions of multivariate stochastic orders are considered, such an extension can be developed along the same lines of the present article. This will be the subject of some future work.

\section{Appendix}

This section is devoted to recalling basic definitions and theorems concerning stochastic dependence and copulas. For further details, see e.g. [13].

We will consider the following positive dependence properties:

Definition 6. $\quad(X, Y)$ is Positive Quadrant Dependent (PQD) if

$$
\bar{F}(x, y) \geq \bar{G}_{X}(x) \bar{G}_{Y}(y) ;
$$

- $Y$ is Right Tail Increasing in $X(\operatorname{RTI}(Y \mid X))$ if $\frac{\bar{F}(x, y)}{\bar{G}_{X}(x)} \uparrow x$;

- $(X, Y)$ is Right Corner Set Increasing (RCSI) if

$$
\bar{F}(x, y) \bar{F}\left(x^{\prime}, y^{\prime}\right) \geq \bar{F}\left(x^{\prime}, y\right) \bar{F}\left(x, y^{\prime}\right)
$$

$\forall x \leq x^{\prime}, y \leq y^{\prime}$, i.e. $\bar{F}$ is Totally Positive of order $2\left(T P_{2}\right)$.

We will consider here only the "joint" absolutely continuous case, with $f$ joint density. We consider then also

Definition 7. $\quad Y$ is Stochastically Increasing in $X(\operatorname{SI}(Y \mid X))$ if

$$
\bar{G}_{Y}(y \mid X=x) \uparrow x ;
$$

- $(X, Y)$ is Positive Likelihood Ratio Dependent (PLRD) if $f$ is $T P_{2}$. 
As known, the dependence properties of a joint distribution are actually properties of its copula only, i.e. they do not involve the margins.

We recall that a copula can be seen as the distribution function of two r.v.'s $U, V$, uniformly distributed on $[0,1]$. In the specific, we considered here the survival copula $\hat{C}(u, v)=\bar{F}\left(\bar{G}_{X}^{-1}(u), \bar{G}_{Y}^{-1}(v)\right)$.

The dependence properties in Definitions 6, 7 can be restated by the following

Definition 8. $\quad(X, Y)$ is PQD if and only if $\hat{C}$ is PQD, i.e. if and only if $\hat{C}(u, v) \geq u v$;

- $\operatorname{RTI}(Y \mid X)$ if and only if $\hat{C}$ is $\operatorname{LTD}(V \mid U)$, i.e. if and only if

$$
\frac{\hat{C}(u, v)}{u} \downarrow u \forall v
$$

- $(X, Y)$ is RCSI if and only if $\hat{C}$ is $\mathrm{TP}_{2}$;

- $\operatorname{SI}(Y \mid X)$ if and only if $\hat{C}$ is $\operatorname{SI}(V \mid U)$, i.e. if and only if

$$
\frac{\partial \hat{C}(u, v)}{\partial u} \downarrow u \forall v ;
$$

- $(X, Y)$ is PLRD if and only if $\frac{\partial^{2} \hat{C}(u, v)}{\partial u \partial v}$ is $\mathrm{TP}_{2}$.

\section{Acknowledgements}

We thank the Editors for inviting us to contribute to this Volume in honour of Moshe Shaked.

\section{References}

[1] E. Arjas, A stochastic process approach to multivariate reliability systems: notions based on conditional stochastic order, Math. Oper. Res. 6 (1981), no. 2, 263-276.

[2] E. Arjas and I. Norros, Life lengths and association: a dynamic approach, Math. Oper. Res. 9 (1984), no. 1, 151-158.

[3] B. Bassan and F. Spizzichino, Relations among univariate aging, bivariate aging and dependence for exchangeable lifetimes, J. Multivariate Anal. 93 (2005), no. 2, 313-339. 
[4] A. Colangelo, T. Hu, and M. Shaked, Conditional orderings and positive dependence, J. Multivariate Anal. 99 (2008), no. 3, 358-371. MR MR2396968 (2008m:60029)

[5] O. Davidov and A. Herman, New tests for stochastic order with application to case control studies, Journal of Statistical Planning and Inference 139 (2009), no. 8, 2614-2623.

[6] _ Multivariate stochastic orders induced by case-control sampling., Methodol. Comput. Appl. Probab. 13 (2011), no. 1, 139-154.

[7] S. Fahmi, C. A. de B. Pereira, F. Proschan, and M. Shaked, The influence of the sample on the posterior distribution, Communications in Statistics - Theory and Methods 11 (1982), no. 16, 1757-1768.

[8] H. Joe, Multivariate models and dependence concepts, Chapman \& Hall, London, 1997.

[9] K. Jogdeo, On a probability bound of Marshall and Olkin, Annals of Statistics 6 (1978), 232-234.

[10] B.-E. Khaledi and S. Kochar, Dependence properties of multivariate mixture distributions and their applications, Annals of the Institute of Statistical Mathematics 53 (2001), no. 3, 620-630.

[11] M.-L. T. Lee, Dependence by total positivity, Annals of Probability 13 (1985), 572-582.

[12] A. J. McNeil, R. Frey, and P. Embrechts, Quantitative Risk Management. Concepts, Techniques and Tools, Princeton Series in Finance, Princeton University Press, Princeton, NJ, 2005.

[13] R. B. Nelsen, An Introduction to Copulas, second ed., Springer Series in Statistics, Springer, New York, 2006.

[14] I. Norros, Systems weakened by failures, Stochastic Processes and their Applications 20 (1985), 181-196.

[15] A. R. Sampson and L. R. Whitaker, Estimation of multivariate distributions under stochastic ordering, Journal of the American Statistical Association 84 (1989), no. 406, 541-548.

[16] M. Shaked and J. G. Shanthikumar, Dynamic multivariate aging notions in reliability theory, Stochastic Processes and their Applications. 
[17] _ Stochastic orders, Springer Series in Statistics, Springer, New York, 2007.

[18] M. Shaked and F. Spizzichino, Positive dependence properties of conditionally independent random lifetimes, Math. Oper. Res. 23 (1998), no. 4, 944-959.

[19] F. Spizzichino, Subjective Probability Models for Lifetimes, Monographs on Statistics and Applied Probability, vol. 91, Chapman \& Hall/CRC, Boca Raton, FL, 2001.

[20] R. Szekli, Stochastic ordering and dependence in applied probability, Lecture Notes in Statistics (Springer). 97. Berlin: Springer-Verlag. viii, 194 p., 1995. 